\title{
INFECCIONES DEL SITIO QUIRÚRGICO POST CRANEOTOMÍAS Y SUS FACTORES DE RIESGOS RELACIONADOS
}

\section{INFECTIONS OF THE POST-CRANIOTOMY SURGICAL SITE AND ITS RELATED RISK FACTORS}

\author{
*Nolbia Yohely Rivera Ferrera, *Paula Liliana Villeda, *Michelle Suazo Padilla, \\ **Elba Odessa Amaya García
}

\section{RESUMEN}

Introducción: A pesar de los avances científicos, la infección post cirugía sigue siendo importante causa de morbilidad y mortalidad. Objetivos: Determinar tasa de incidencia, analizar factores de riesgo y caracterizar las infecciones del sitio quirúrgico (ISQ). Pacientes y métodos: Estudio cuantitativo, descriptivo, correlacional, retrospectivo de corte transversal, de 117 expedientes de pacientes con craneotomías limpias, que reunieron los criterios de inclusión del servicio de neurocirugía de adulto del Hospital Mario Catarino Rivas de junio del 2016 a mayo del 2017. Resultados: La tasa de incidencia de ISQ fue del $11,97 \%$, que ocupó el primer lugar en incidencia junto con las infecciones de origen pulmonar. Las ISQ en heridas limpias fueron $10 \%$ y $30 \%$ en heridas con cuerpo extraño. Los agentes etiológicos más frecuentes fueron los bacilos gramnegativos. Los principales factores de riesgo intrínsecos fueron el sexo femenino ([OR] Odds Ratio=3), clasificación ASA I $(\mathrm{OR}=4.4)$ y la patología tumoral $(\mathrm{OR}=3.3)$; los extrínsecos fueron, hospitalización de más de 30 días $(\mathrm{OR}=5.4)$, re-intervenciones $(\mathrm{OR}=3.8)$, fuga de Liquido Cefalorraquideo (LCR) (OR=136), transfusión sanguínea $(\mathrm{OR}=12.6)$, catéter venoso central $(\mathrm{OR}=5.3)$, catéter vesical más de 72 horas $(\mathrm{OR}=6.7)$, traqueotomía $(\mathrm{OR}=4.2)$, injerto dural $(O R=6.5)$ y sin drenaje postquirúrgico $(\mathrm{OR}=4.6)$. Conclusión: La incidencia

*Doctora en Medicina y Cirugía. (UNAH-VS)

**Doctora en Cirugía Dental. (UNAH-VS)

Dirigir correspondencia a:

yoherivera@gmail.com, https://orcid.org/0000-0002-8793-8397;

paularivas_4@hotmail.com, https://orcid.org/0000-0003-1875-9405;

michel_8814@hotmail.com,https://orcid.org/0000-0002-6111-7590;

drelba.amaya@gmail.com, https://orcid.org/0000-0002-8377-5025

Recibido: 31 de enero del 2019 Aprobado: 30 de octubre del 2019 de ISQ fue alta, con mayor proporción en mujeres y en edad de 31 a 40 años. Tres factores de riesgo intrínsecos y nueve extrínsecos se relacionaron significativamente con ISQ, revelando mayor número de factores modificables.

\section{PALABRAS CLAVE}

Craneotomía, Factores de Riesgo, Infección de la Herida quirúrgica.

\section{ABSTRACT}

Introduction: Despite scientific advances, infection after surgery remains an important cause of morbidity and mortality. Objectives: To determine incidence rate, analyze risk factors and characterize surgical site infections (SSI). Patients and methods: Quantitative, descriptive, correlational, retrospective cross-sectional study of 117 records of patients with clean craniotomies, who met the inclusion criteria of the adult neurosurgery service of Mario Catarino Rivas Hospital between June 2016 to May 2017. Results: The incidence rate of SSI was $11.97 \%$, which ranked first in incidence along with infections of pulmonary origin. The SSI in clean wounds were $10 \%$ and $30 \%$ in wounds with a foreign body. The most frequent etiologic agents were gram-negative bacilli. The main intrinsic risk factors were female sex ([OR] Odds Ratio=3), ASA I classification $(\mathrm{OR}=4.4)$ and tumor pathology $(\mathrm{OR}=3.3)$; the extrinsics were more than 30 days of hospitalization $(\mathrm{OR}=5.4)$, re-interventions $(\mathrm{OR}=3.8), \mathrm{CSF}$ leakage $(\mathrm{OR}=136)$, blood transfusion $(\mathrm{OR}=12.6)$, central venous catheter $(\mathrm{OR}=5.3)$, Bladder catheter more than 72 hours $(\mathrm{OR}=6.7)$, tracheotomy $(\mathrm{OR}=4.2)$, 
dural graft $(\mathrm{OR}=6.5)$ and without post-surgical drainage $(\mathrm{OR}=4.6)$. Conclusions: The incidence of SSI was high, with a higher proportion in women between 31 to 40 years old. Three intrinsic and nine extrinsic risk factor were significantly related to ISS, revealing a greater number of modifiable factors.

\section{KEYWORDS}

Craniotomy, Risk Factors, Surgical Wound Infection.

\section{INTRODUCCIÓN}

Antes de la antisepsia quirúrgica de Lister (1867), casi el $80 \%$ de las operaciones fueron seguidas por infecciones en el sitio quirúrgico (ISQ) y casi la mitad murieron. A pesar de los avances científicos, la infección post cirugía sigue siendo importante causa de morbilidad y mortalidad. (1) Además ocasiona costos evitables ligados a la prolongación de la estadía hospitalaria, re intervenciones quirúrgicas, antibióticos y estudios costosos. $^{(2)}$

La infección del tracto urinario $(40 \%)$, y la ISQ $(24 \%)$ son las infecciones nocosomiales más frecuentes, las cuales se definen como las que aparecen 48 horas después del ingreso y hasta 72 horas después del alta. ${ }^{(3,4)}$ Las tasas de ISQ van de menos del $1 \%$ a $5 \%$ en heridas limpias con profilaxis antibiótica, y de $10 \%$ a $20 \%$ en heridas sucias. ${ }^{(1,5-7)}$ Las heridas limpias en neurocirugía se subdividen en aquellas con o sin la implantación de derivación ventricular externa (DVE). Los Centros para el Control y Prevención de enfermedades (CDC, [Centers for Disease Control and Prevention]) definen las infecciones incisionales superficiales, las que son limitadas a la piel y al tejido subcutáneo $(60 \%)$; las profundas, al espacio subgaleal y el colgajo óseo; y las de órgano corresponden a la meningitis (22\%), el empiema subdural y el absceso cerebral $(14 \%){ }^{(1)}$
Los factores de riesgo implicados en desarrollar una ISQ post craneotomía, se pueden dividir para un mejor análisis, en intrínsecos (relacionados con el paciente) y extrínsecos (relacionados con la cirugía y el ambiente hospitalario). ${ }^{(1,3,4)}$ La profilaxis correcta, requiere el uso del antibiótico adecuado, con inicio de 30 a 60 minutos antes de la incisión quirúrgica y en caso que la duración de una cirugía exceda las 4 horas, se debe infundir una dosis adicional. ${ }^{(1,6,8)} \mathrm{El}$ tiempo (T), es el percentil 75 de la duración de un procedimiento dado (CDC), donde la herida quirúrgica está expuesta a contaminación potencial. Para craneotomías, el tiempo (T) es de 4 horas. ${ }^{(6,7,9)}$ La fiebre postoperatoria se relaciona con infección, sin embargo es probable que su causa entre los 3 días siguientes a la operación sea no infecciosa. ${ }^{(7)}$

Ya que los datos disponibles de esta problemática son escasos en la literatura nacional; motivó este estudio con el objetivo de determinar la tasa de incidencia de ISQ, analizar su relación con los principales factores de riesgo y caracterizar las ISQ en pacientes sometidos a craneotomías del servicio de neurocirugía de adultos en el Hospital Nacional Mario Catarino Rivas, para contribuir con evidencia que permitan implementar medidas de prevención y ofrecer una atención de calidad.

\section{PACIENTES Y METODOS}

Se realizó un estudio cuantitativo, descriptivo, correlacional y retrospectivo de corte transversal sobre la relación entre las ISQ post craneotomías y sus factores de riesgo, en el servicio de neurocirugía de adulto del Hospital Mario Catarino Rivas de San Pedro Sula, Cortés, Honduras; en el período de junio del 2016 a mayo del 2017. La población y muestra fueron los 117 expedientes de pacientes con craneotomías limpias con o sin derivación ventricular externa (DVE) que reunieron los criterios de inclusión para su análisis. Se extrajo el listado de craneotomías de los libros quirúrgicos del departamento de anestesia. 


\section{Criterios de inclusión}

1. Hombres y mujeres mayores de 18 años.

2. Craneotomías con herida quirúrgica de clase limpia según la clasificación de Altemeier (CDC), ${ }^{(1,6,7)}$ que incluye incisiones primitivamente cerradas, en la cual no se efectuó invasión quirúrgica del tracto respiratorio, tubo digestivo y genitourinario.

3. Heridas con cuerpo extraño como ser derivación ventricular externo, monitoreo de presión intracraneal y drenajes post quirúrgicos.

4. Pacientes con trauma cerebral.

5. Desenlace de la infección hasta 30 días post quirúrgico y hasta un año para implante protésico según registros disponibles por consulta externa.

\section{Criterios de Exclusión}

1. Pacientes con traumas penetrantes o con laceraciones.

2. Con procesos infecciosos que ya estaban con esquema antibiótico pre cirugía.

3. Craneotomías en las que se estableció la infección al momento de la cirugía, como ser abscesos cerebrales, empiema o meningitis.

4. Cirugías con abordaje trans esfenoidal.

5. Pacientes post quirúrgicos que murieron antes de los treinta días por causa diferente a infección nosocomial.

6. Derivaciones ventrículo peritoneal, que a pesar de ser heridas quirúrgicas limpias, presentan alto riesgo de infección.

7. Re-intervenciones en lapsos menores de 30 días.

8. Pacientes trasladados hacia otro centro médico y expedientes incompletos.

Los datos se recolectaron por medio de un cuestionario estructurado. La fuente de información fue el expediente clínico tomando referencia las notas quirúrgicas de neurocirugía y anestesia; registros diarios de médicos, enfermería y estudios de laboratorio. El diagnóstico de infección se realizó siguiendo los criterios de los CDC. ${ }^{(6,8)}$
Se evaluaron factores de riesgo intrínseco como ser: sexo, edad, urgencia de la intervención, comorbilidad, causa de la cirugía, puntaje de Glasgow al ingreso y el índice de la Sociedad Americana de Anestesiología (ASA, [American Society of Anesthesiology]) evaluada por el anestesiólogo.

Los factores de riesgo extrínseco fueron: tiempo de estadía pre y post cirugía en sala de hospitalización, sala de recuperación y unidad de cuidados intensivos (UCl); duración en horas de la cirugía; técnicas y procedimientos peri operatorio como el uso de ventilación mecánica, sonda vesical, catéter venoso central (CVC), drenaje postoperatorio de la herida, derivación ventricular externa, implantes protésicos, fuga de líquido cefalorraquídeo (LCR), re intervenciones y transfusión sanguínea. Los antibióticos administrados 2 horas antes de la cirugía se consideraron profilácticos y los usados post quirúrgico como terapéuticos. ${ }^{(8)}$

La base de datos y el análisis estadísticos se realizó en Microsoft Office Excel 2007. Se usó la incidencia como medida de frecuencia de la enfermedad. Las variables entre el grupo de casos de 14 pacientes con ISQ y el grupo control de 103 pacientes sin ISQ, se compararon mediante la prueba Chi cuadrado (x2) para las variables categóricas; y la prueba Z se utilizó para las variables continuas. La significancia estadística se definió como un valor $p<0.05$ para determinar la presencia de una asociación entre dos variables. La fuerza de asociación se midió con Odds Ratio (OR); y se calcularon intervalos de confianza (IC) del 95\%.

El desarrollo de este estudio cumplió con las pautas éticas para la investigación con seres humanos. El protocolo fue autorizado por el servicio de Docencia e Investigación, Dirección General del Hospital y servicio de Neurocirugía. No se requirió de consentimiento informado ya que únicamente se revisaron expedientes clínicos, declarando 
que no aparecen datos de pacientes, conservando así el derecho a la privacidad.

\section{RESULTADOS}

Un total de 117 craneotomías reunieron los criterios de inclusión para su análisis.
Se encontró una tasa de incidencia de ISQ de $11.97 \%(n=14 / 117)$, que ocupó el primer lugar, al igual que las infecciones de origen pulmonar y el $35 \%(n=5 / 14)$ de las ISQ se presentaron concomitantemente con otro tipo de Infecciones nosocomiales. (Ver Tabla No. 1).

Tabla No. 1: Frecuencia e Incidencia de los diferentes tipos de Infecciones que se presentaron concomitantemente con las ISQ.

\begin{tabular}{|c|c|c|c|c|c|c|c|}
\hline Descripción & $\mathbf{N}^{*}$ & $\%$ & ISQ † & Pulmonar & Urinaria & CVC $\neq$ & Otros \\
\hline ISQ & 9 & 8 & 9 & & & & \\
\hline ISQ + Otros & 5 & 4 & 5 & 2 & 3 & & \\
\hline Otros & 18 & 15 & & 12 & 5 & 2 & 3 \\
\hline Ninguna & 85 & 73 & & & & & \\
\hline Total & 117 & $100 \%$ & 14 & 14 & 8 & 2 & 3 \\
\hline \multicolumn{3}{|c|}{ Tasas de Incidencia } & $11.97 \%$ & $11.97 \%$ & $7 \%$ & $2 \%$ & $3 \%$ \\
\hline
\end{tabular}

Fuente: Expedientes clínicos de pacientes con Craneotomías del Hospital Mario Catarino Rivas.

${ }^{*} \mathrm{~N}$, número de frecuencia; †ISQ, Infecciones del Sitio quirúrgico; $¥$ CVC, Catéter Venoso Central.

De los tipos de ISQ, las más frecuentes fueron de superficie con $64.3 \%(n=9 / 14)$ de las cuales el $67 \%(n=6 / 9)$ desarrolló extensión profunda al hueso o cavidad intracraneal; produciendo al final un $57 \%(n=8 / 14)$ de infección en órgano/espacio y del $35.7 \%$ $(n=5 / 14)$ en las profundas (espacio subgaleal/óseo). Dentro de las infecciones del sistema nervioso central, la meningitis y el empiema epidural-subdural tuvieron mayor porcentaje con un $38 \%$ cada una $(n=3 / 8$ pacientes) y el absceso cerebral en segundo lugar con el $25 \%(n=2 / 8)$.
Geográficamente el mayor porcentaje de pacientes procedían de los departamentos de Cortés 40\% (47/117), Santa Bárbara 17\% $(n=20 / 117)$, Yoro $13 \%(n=15 / 117)$, Atlántida $9 \%(n=10 / 117)$, otros $21 \%$. El $50 \%(n=7 / 14)$ de los casos con ISQ se presentó en pacientes que provenían de Cortés. La población se caracterizó por ser el $66 \%$ hombres y la edad más frecuente mayor de 70 años (media 59.4 \pm 18 años).

En la Tabla No. 2 se presentan los factores de riesgo intrínsecos, dentro de los cuales el sexo mujer, ASA I y los pacientes con patología tumoral, específicamente Meningiomas, tuvieron relación estadísticamente significativa con ISQ. 
Tabla No. 2: Factores de riesgo intrínsecos asociados a ISQ.

\begin{tabular}{|c|c|c|c|c|c|c|c|c|}
\hline \multirow{2}{*}{\multicolumn{2}{|c|}{ Factor de Riesgo }} & \multirow{3}{*}{$\begin{array}{r}\% \\
66\end{array}$} & \multirow{3}{*}{$\begin{array}{c}\begin{array}{c}\text { Incidencia } \\
\text { ISQ* }^{*}\end{array} \\
8\end{array}$} & \multicolumn{2}{|c|}{ IC $95 \%$} & \multirow{3}{*}{$\frac{x^{2}}{3.72}$} & \multirow{3}{*}{$\begin{array}{c}\text { OR } \\
0.3\end{array}$} & \multirow{3}{*}{$\begin{array}{c}\boldsymbol{P} \\
\mathrm{Ns}+\end{array}$} \\
\hline & & & & \multirow{2}{*}{$\begin{array}{l}\text { L. Inf. } \\
2.9\end{array}$} & \multirow{2}{*}{$\frac{12.7}{12.7}$} & & & \\
\hline Sexo & Hombre & & & & & & & \\
\hline & Mujer & 34 & 20 & 12.8 & 27.2 & 3.8 & 3 & 0.05 \\
\hline \multirow{3}{*}{ (años) } & $>70$ & 31 & 3 & -0.2 & 5.8 & 4.2 & 0.1 & $<0.05$ \\
\hline & $31-40$ & 15 & 24 & 15.8 & 31.2 & 2.5 & 2.8 & Ns \\
\hline & $18-30$ & 6 & 14 & 8.3 & 20.3 & 0 & 1.2 & \\
\hline \multicolumn{9}{|c|}{ Comorbilidad } \\
\hline \multicolumn{2}{|c|}{$\mathrm{DM}+\mathrm{HTA}+$ Otros $\ddagger$} & 12 & 21 & 14 & 28.9 & 1.4 & 2.3 & Ns \\
\hline \multicolumn{2}{|r|}{ DM } & 3 & 0 & 0 & 0 & 0.6 & 0 & Ns \\
\hline \multicolumn{2}{|r|}{ HTA + Otros } & 24 & 7 & 2.5 & 11.8 & 0.8 & 0.5 & \\
\hline \multicolumn{2}{|r|}{ Otros } & 13 & 18 & 11.2 & 25.2 & 0.4 & 1.7 & \\
\hline \multicolumn{2}{|r|}{ Ninguna } & 51 & 12 & 5.8 & 17.5 & 0 & 0.9 & Ns \\
\hline \multirow[t]{5}{*}{$A S A^{* *}$} & Clase I & 8 & 33 & 24.8 & 41.9 & 4.2 & 4.4 & $<0.05$ \\
\hline & Clase II & 15 & 0 & 0 & 0 & 2.7 & 0 & Ns \\
\hline & Clase III & 18 & 19 & 11.9 & 26.2 & 1.2 & 2 & Ns \\
\hline & Clase IV & 9 & 9 & 3.9 & 14.3 & 0.1 & 0.7 & \\
\hline & Sin Clasificar & 50 & 10 & 4.7 & 15.6 & 0.4 & 0.7 & Ns \\
\hline \multicolumn{9}{|c|}{ Glasgow al Ingreso } \\
\hline \multicolumn{2}{|r|}{$15-13$} & 72 & 11 & 5.1 & 16.3 & 0.4 & 0.7 & \\
\hline \multicolumn{2}{|r|}{$12-9$} & 15 & 11 & 5.4 & 16.8 & 0 & 0.9 & \\
\hline \multicolumn{2}{|c|}{ Igual $0<8$} & 13 & 20 & 12.8 & 27.2 & 1.1 & 2.1 & Ns \\
\hline \multicolumn{9}{|c|}{ Tipo de Cirugía } \\
\hline \multicolumn{2}{|c|}{ Electiva } & 24 & 18 & 10.9 & 24.8 & 1.2 & 1.9 & Ns \\
\hline \multicolumn{2}{|c|}{ Urgente } & 76 & 10 & 4.6 & 15.6 & 1.2 & 0.5 & \\
\hline \multicolumn{9}{|c|}{ Causa de la Cirugía } \\
\hline \multirow{2}{*}{\multicolumn{2}{|c|}{$\begin{array}{c}\text { Hemorrag. Intracraneal } \\
\text { Tumores }\end{array}$}} & 74 & 8 & 3.2 & 13.1 & 39.4 & 0.3 & $<0.001$ \\
\hline & & 26 & 23 & 15 & 30.2 & 39.4 & 3.3 & $<0.001$ \\
\hline Diagnó & tico & & & & & & & \\
\hline $\mathrm{He}$ & natoma Epidural & 9 & 0 & 0 & 0 & 1.5 & 0 & \\
\hline Her & natoma Subdural & 50 & 5 & 1.1 & 9.1 & 5.3 & 0.2 & $<0.05$ \\
\hline & HSA†† & 8 & 22 & 14.7 & 29.8 & 1 & 2.3 & \\
\hline Hemc & rrag. Parénquima & 7 & 25 & 17.2 & 32.8 & 1.4 & 2.7 & \\
\hline & Meningiomas & 11 & 31 & 22.4 & 39.1 & 4.9 & 4.2 & $<0.05$ \\
\hline Glic & mas Bajo grado & 6 & 0 & 0 & 0 & 1 & 0 & \\
\hline Gli & omas Alto grado & 3 & 0 & 0 & 0 & 0.6 & 0 & \\
\hline & tros Tumores & 6 & 43 & 33.9 & 51.8 & 6.7 & 6.8 & $<0.05$ \\
\hline
\end{tabular}

Fuente: Expedientes clínicos de pacientes con Craneotomías del Hospital Mario Catarino Rivas.

*Los datos representan la Tasa de Incidencia de Infecciones del Sitio Quirúrgico; †Ns, no significativo; ¥ DM, Diabetes Mellitus. HTA, Hipertención Arterial; ${ }^{* *}$ ASA, American Society of Anesthesiologists; ††HSA, Hemorragia Subaracnoidea. 
Los factores de riesgo extrínseco, fueron caracterizados según el tiempo de estadía en las diferentes salas hospitalarias y pabe- llones quirúrgicos (Tabla No. 3); y según los procedimientos perioperatorios (Tabla No. 4).

Tabla No. 3: Factores de riesgo extrínsecos asociados a ISQ, según la duración de la estadía en las diferentes salas.

\begin{tabular}{|c|c|c|c|c|c|c|c|c|c|c|}
\hline \multirow{3}{*}{$\begin{array}{c}\text { Factor de Riesgo } \\
\text { Estadía Pre cirugía }\end{array}$} & \multirow{3}{*}{$\%$} & \multirow{2}{*}{ ISQ (IC 95\%)* } & \multirow{2}{*}{$x^{2}$} & \multirow{2}{*}{ OR } & \multirow{2}{*}{$P$} & \multicolumn{5}{|c|}{ Valores Medio } \\
\hline & & & & & & Rango & $x$ & Casot & control† & - $Z$ \\
\hline & & & & & & $<1$ a 65 & 7.3 & 12 & 6.7 & 95 \\
\hline Menos de 1 (días) & 28 & $9(3.9-14.3)$ & 0.4 & 0.7 & & días & días & días & días & \\
\hline 1 a 10 & 51 & $12(5.8-17.5)$ & 0 & 0.9 & & & & & & \\
\hline Más de 10 & 21 & $17(9.9-23.4)$ & 0.6 & 1.7 & Ns & & & & & \\
\hline Estadía Pos cirugía & & & & & & 2 a 76 & 11.5 & 36 & 8.2 & 03 \\
\hline 1 a 3 (días) & 18 & $5(0.8-8.3)$ & 1.4 & 0.3 & & días & días & días & días & \\
\hline 4 a 10 & 56 & $2(-0.7-3.8)$ & 15.1 & 0 & $<0.001$ & & & & & \\
\hline 11 a 30 & 14 & $13(6.5-19)$ & 0.0 & 1.1 & & & & & & \\
\hline Más de 30 & 12 & $71(63-79.6)$ & 30.8 & 21.6 & $<0.001$ & & & & & \\
\hline Hospitalización Total & & & & & & 3 a 95 & 19 & 48 & 15 & 68 \\
\hline 1 a 5 (días) & 29 & & 6.5 & 0 & $<0.05$ & días & días & días & días & \\
\hline 6 a 15 & 32 & $3(-0.2-5.6)$ & 4.4 & 0.1 & $<0.05$ & & & & & \\
\hline Más de 30 & 25 & $38(29-46.7)$ & 9.3 & 5.4 & $<0.01$ & & & & & \\
\hline No consignados & 14 & & & & Ns & & & & & \\
\hline Duración de la Cirugía & & & & & & $15 \min a$ & 2 & 2.8 & 1.8 & $9^{n}$ \\
\hline$<1$ (horas) & 56 & $6(1.8-10.5)$ & 4.7 & 0.3 & $<0.05$ & 9 hras & hras & hras & hras & \\
\hline $1.1-2$ & 12 & $21(14-28.9)$ & 1.4 & 2.3 & & & & & & \\
\hline$>4$ & 9 & $27(19-35.3)$ & 2.7 & 3.2 & Ns & & & & & \\
\hline No consignados & 23 & & & & Ns & & & & & \\
\hline Sala de Recuperación & & & & & & $1 \mathrm{hra}$ a & 70 & 185 & 56 & .4 \\
\hline 1 a 4 (horas) & 11 & $8(3-12.5)$ & 0.3 & 0.6 & & 47 días & Hras & hras & hras & \\
\hline Más de 48 & 30 & $23(15.7-31)$ & 4.9 & 3.5 & $<0.05$ & & & & & \\
\hline No consignados & 59 & & & & Ns & & & & & \\
\hline Estadía en UCl & & & & & & 2 a 22 & 11 & 12.5 & 10.7 & 29 \\
\hline Sin Uso de UCI & 93 & $11(5-16.7)$ & 1.4 & 0.4 & Ns & días & días & días & días & \\
\hline Uso De UCl & 7 & $25(17-33)$ & 1.4 & 2.7 & Ns & & & & & \\
\hline
\end{tabular}

Fuente: Expedientes Clínicos de pacientes con Craneotomías del Hospital Mario Catarino Rivas.

* Tasa de Incidencia de ISQ con sus respectivos IC del 95\%; †Datos de la media de casos con ISQ y controles sin ISQ. 
Tabla No. 4: Factores de riesgo extrínsecos según el procedimiento peri operatorio, asociados a ISQ.

\begin{tabular}{|c|c|c|c|c|c|}
\hline Factor de Riesgo & $\%$ & ISQ (IC95\%) & $x^{2}$ & OR & $P$ \\
\hline Ventilación Mecánica & 18 & $29(21$ - 37.6) & 5.7 & 4.2 & $<0.05$ \\
\hline Catéter Venoso Central & 7 & $38(28.7-46)$ & 5.3 & 5.3 & $<0.05$ \\
\hline Ninguno & 75 & - & - & - & - \\
\hline \multicolumn{6}{|l|}{ Profilaxis Antibiótica } \\
\hline Ninguna & 6 & & 0.4 & 0.0 & \\
\hline Ceftriaxona (1) & 38 & $7(2.3-11)$ & 1.8 & 0.4 & \\
\hline Terapia Post-Cirugía & 56 & $17(10-23.7)$ & 3.4 & 3.3 & Ns \\
\hline No consignados & 3 & & & & Ns \\
\hline \multicolumn{6}{|l|}{ Implante Protésico } \\
\hline Injerto dural & 9 & $40(31-49)$ & 8.2 & 6.5 & $<0.01$ \\
\hline Transfusión Sanguínea & 23 & $37(28-45.8)$ & 20.9 & 12.6 & $<0.001$ \\
\hline Ninguno & 68 & - & - & - & - \\
\hline \multicolumn{6}{|l|}{ Sonda Urinaria } \\
\hline Menos de 72 horas & 32 & $5(1.3-9.5)$ & 2.2 & 0.5 & Ns \\
\hline Más de 72 horas & 32 & $26(18.3-34)$ & 11 & 6.7 & $<0.001$ \\
\hline No usó & 36 & $5(0.9-8.6)$ & 3.2 & 0.3 & Ns \\
\hline \multicolumn{6}{|l|}{ Drenaje Post- Operatorio } \\
\hline Con Drenaje & 59 & $3(-0.1-6)$ & 13.1 & 0.1 & $<0.001$ \\
\hline $\mathrm{DVE}^{*}$ & 9 & $30(21.7-38)$ & 3.4 & 3.7 & Ns \\
\hline Sin Drenaje & 32 & $24(16-31.4)$ & 7.3 & 4.6 & $<0.01$ \\
\hline Re intervención & 15 & $28(19.7-36)$ & 5 & 3.8 & $<0.05$ \\
\hline Salida de LCR† & 8 & $89(83-94.6)$ & 54.8 & 136 & $<0.001$ \\
\hline \multicolumn{6}{|l|}{ Tipo de Herida } \\
\hline Limpia & 91 & $10(4.7-15.8)$ & 3.4 & 0.3 & Ns \\
\hline Con Cuerpo extraño & 9 & $30(21.6-38)$ & 3.4 & 3.7 & Ns \\
\hline
\end{tabular}

Fuente: Expedientes Clínicos de pacientes con Craneotomías del Hospital Mario Catarino Rivas.

*DVE, Derivación Ventricular Externo; †LCR, Líquido Cefalorraquídeo.

El $79 \%(n=11 / 14)$ de las ISQ poseían cultivo de secreciones. Los agentes etiológicos detectados según el sitio de infección se presentan en la Tabla No. 5. 
Tabla No. 5: Frecuencia de agentes etiológicos detectados en ISQ post craneotomías.

\begin{tabular}{l|c}
\hline Organismo Causal & ISQ \\
\hline \hline Psudomonas SPP* & 6 \\
Klepsiella Pn. & 4 \\
Acinetobacter Spp & 2 \\
Staphilococos A (SARM)† & 1 \\
Acinetobacter Boumanii & 1 \\
Enterobacter Cloacae* & 1 \\
Serratia Spp & 1 \\
Sin Evidencia & 3 \\
\hline \hline
\end{tabular}

Fuente: Información extraída de los resultados de cultivos de ISQ en pacientes con craneotomías del Hospital Mario Catarino Rivas.

*Un caso de Multiresistencia, †SARM: Staphilococos Aureus Meticilino Resistente.

Los casos con ISQ se caracterizaron por presentar fiebre y leucocitosis en el $79 \%$, con un tiempo medio en manifestarse la infección de $14.1 \pm 6.5$ días, (rango de 5 a 30 días) y el $28.5 \% \quad(n=4 / 14)$ de ellos se diagnosticaron posterior al alta. El tratamiento de las infecciones fue quirúrgico en el $50 \%$ de los casos $(n=7 / 14) ; y$ el promedio del número de días de hospitalización posterior a las infecciones fue de 34 días (rango de 7 a 90 días). Se encontró una tasa de mortalidad de las ISQ del $3.4 \%(4 / 117)$ y de letalidad del 28\% (4/14).

\section{DISCUSION}

A la luz de los datos, nuestro trabajo obtuvo cifras de incidencias más altas de ISQ en heridas limpias (10\%) y con cuerpo extraño (30\%). En comparación con la bibliografía revisada, donde reportaron tasas de ISQ en

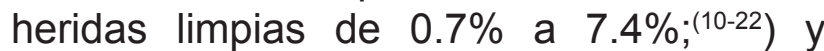
según Lietard et al ${ }^{(16)}$ (Francia) y Martínez-Checa et al ${ }^{(19)}$ (España), las tasas de incidencia en heridas con cuerpo extraño fueron menos del $10 \%$. Sin embargo, Bellusse et al ${ }^{(23)}$ (Brasil), mostraron una incidencia de ISQ en heridas limpias similar a la nuestra (9.4\%); por otro lado Perdomo-Sabillón et al, ${ }^{(24)}$ en un estudio realizado en el Hospital Escuela, Tegucigalpa, reportaron una tasa de ISQ en heridas limpias más alta $(12.8 \%)$.

Con respecto a los tipos de ISQ, obtuvimos alto porcentaje de ISQ en órgano/espacio $(57 \%)$ después de las superficiales, a pesar de ser considerada la menos frecuente y más alarmante. ${ }^{(10,14,15)}$ En consecuencia, en nuestro estudio la incidencia de meningitis se elevó hasta $2.6 \%$, en comparación con la incidencia de $1.5 \%$ reportada por Korinek et $\mathrm{al}^{(17)}$ en Francia.

Geográficamente, nuestros pacientes procedían de 11 de las 18 regiones del país, principalmente del departamento de Cortés, evidenciándose que el Hospital Mario Catarino Rivas es un centro médico de importante relevancia en Honduras. La población en riesgo de presentar ISQ se caracterizó por ser más frecuente en mujeres y en el grupo de edad joven (31-40 años, OR=2.8, ns); donde los pacientes mayores de 70 años fueron menos afectados $(O R=0.1 ; p<0.05)$, que podría ser por el trato multidisciplinario hacia este grupo, por ser considerados de alto riesgo. ${ }^{(20)}$ En cambio Petric ${ }^{(15)}$ y Bellusse, ${ }^{(23)}$ reportaron predominio de ISQ en mayores de 60 años.

Entre los factores de riesgo intrínsecos significativos, afín con dos estudios en España, ${ }^{(20,25)}$ la patología tumoral fué asociada a ISQ, ya que estos pacientes son inmunológicamente susceptibles. En cambio, Petric et al ${ }^{(15)}$ (Romania), Sánchez-Arenas et al (22) (México) y otros, ${ }^{(13,16,19,20)}$ asociaron las ISQ con un ASA mayor a II; a diferencia de nuestro resultado que se asoció con ASA I, que podría ser por la mayor relación de ISQ con el rango de edad joven y a la ausencia de una clasificación ASA en el $50 \%$ de los expedientes. 
El resto de factores intrínsecos destacados (aunque no significativos), fueron el estado de conciencia alterado y comorbilidad, especialmente Hipertensión Arterial (HTA) y Diabetes Mellitus (DM), al igual que en los trabajos de Chisbert-Genovés( ${ }^{(20)}$ y Bellusse. ${ }^{(23)}$ Por otro lado, Jiang et al ${ }^{(13)}$ (China) y Buffet-Bataillona et al (18) (Francia), reportaron que las cirugías de carácter urgente fueron menos frecuente (15 a 38\%) y la más asociada a ISQ. En cambio, en nuestro estudio, la cirugía electiva fue la menos frecuente $(24 \%)$, y con mayor incidencia de ISQ ( $\mathrm{OR}=1.9 ; \mathrm{ns}$ ), que podría deberse a la relación de este tipo de cirugías con factores como tumores, comorbilidad y hospitalizaciones prolongadas.

Dentro de nuestros factores de riesgo extrínsecos significativos, las estadía prolongadas post cirugía en las diferentes salas fueron asociadas con ISQ, al igual que en los estudios de Petric ${ }^{(15)}$ y Bellusse. ${ }^{(23)}$ También fueron significativos en concordancia con otros autores, las re-intervenciones, ${ }^{(12,16,19,21,22)}$ fuga de $\operatorname{LCR}^{(12,17,20,21)}$ y la transfusión sanguínea;(23) Múñez et al, ${ }^{(25)}$ tambien asociaron la presencia de catéter central $(31 \%)$, sonda vesical $(39 \%)$ y ventilación mecánica (11\%); y Petric ${ }^{(15)}$ con la colocación de cuerpos extraños.

Según Augello-Díaz et al(4) (Cuba) y Taha et al(12) (Egipto), los drenajes post quirúrgicos aumentan el riesgo de ISQ. Sin embargo, nuestro resultado fue poco común, con mayor riesgo en quienes no usaron drenaje $(\mathrm{OR}=4.6 ; p<0.01)$, a diferencia de los que si usaron ( $O R=0.1 ; p<0.001)$. Según Mangram

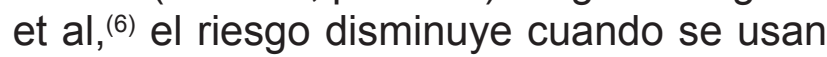
drenajes de succión cerrados que pueden evacuar efectivamente los hematomas o seromas postoperatorios.

Los otros factores extrínsecos, como tiempo de cirugía más de 4 horas $(O R=3.2)$, ingreso a $\mathrm{UCl}(\mathrm{OR}=2.7)$ y profilaxis antibiótica de inicio inadecuado $(\mathrm{OR}=3.3$ - aunque no significativos, también se asociaron a ISQ, al igual que en los trabajos de Lietard et al ${ }^{(16)}$ y Chiang et al. ${ }^{(21)}$ No obstante, la profilaxis antibiótica se inició adecuadamente sólo en el $38 \%$ de nuestros pacientes, con baja incidencia de ISQ del $7 \%(\mathrm{OR}=0.4 ; n s)$. Petric et al, ${ }^{(15)}$ relacionaron el uso de antibiótico post cirugía con la ISQ $(\mathrm{OR}=2.703 ; \mathrm{p}=0.001)$. Palacios-Saucedo et al(26) (México), reportaron que el $96 \%$ de la profilaxis antibiótica no se inició adecuadamente, donde su uso prolongado ponía al paciente en riesgo de ISQ por gérmenes multi resistentes. Korinek et al, ${ }^{(17)}$ mostraron que la profilaxis antibiótica redujo las ISQ de una tasa del $8.8 \%$ hasta el 4.6\% (p 0.0001). Según la Guía para la prevención de ISQ, (CDC, 2017), ${ }^{(8)}$ recomiendan que para procedimientos limpios, no administrar antibióticos post cirugía, incluso en presencia de un drenaje (Categoría IA).

Al comparar la caracterización de los casos, Buang et $a^{(14)}$ (Malasia) y Chiang et al ${ }^{(21)}$ (EEUU), reportaron porcentajes más bajos que los nuestros de fiebre $(26$ a $51 \%$ ) y leucocitosis (58 a $65 \%$ ), motivo por el cual recomendaron la velocidad de sedimentación globular y la proteína $\mathrm{C}$ reactiva como parámetros más sensibles. Para Ducel et $\mathrm{al}^{(2)}(\mathrm{OMS})$, el promedio de días de hospitalización post infección fue más corto $(8,2$ días, rango 3 a 20 días). Por otro lado, Taha ${ }^{(12)}$ y Chisbert-Genovés, ${ }^{(20)}$ mostraron cifras más altas de casos que requirieron limpieza quirúrgica (68 a $78 \%$ ). Jiang ${ }^{(13)}$ y Chiang, ${ }^{(21)}$ revelaron alto porcentajes de pacientes diagnosticados con ISQ después del alta (68 a 70\%); sin embargo Taha ${ }^{(12)}$ reportó una tasa de $20.5 \%$, similar a la nuestra, esto puede deberse a que la mayoría de infecciones superficiales son tratadas ambulatoriamente. Nuestro tiempo promedio en detectar la ISQ (14.1 \pm 6.5 días), se encuentra dentro del rango encontrado en la bibliografía que varió de 11.8 a 66.4 días. ${ }^{(10,}$ $14,20,21)$ 
Los agentes etiológicos mas frecuentes en nuestro estudio fueron los bacilos gramnegativos, principalmente Pseudomona Aureginosa; contrario a otros trabajos, que predominaron los cocos grampositivos, particularmente el Staphylococcus aureus. $(9,12,15,19-25)$

Chisbert-Genovés ${ }^{(20)}$ mostraron un porcentaje de ISQ superficiales que desarrollaron extensión profunda más alto $(92.8 \%)$ que el nuestro $(67 \%)$ y con tasa de letalidad más baja $(21.4 \%)$ que la nuestra (28.6\%). Taha ${ }^{(12)}$ y Petric ${ }^{(15)}$ reportaron una tasa de letalidad atribuible a ISQ de $4.8 \%$.

Entre las limitaciones, encontramos que por ser este estudio de tipo retrospectivo, no cuenta con ciertas variables peri operatoria que hubieran sido de interés; también se presentaron obstáculos para el acceso a los expedientes clínicos, que prolongó el tiempo de este estudio. Sin embargo nuestros resultados ofrecen evidencia que permita orientar otras investigaciones futuras.

\section{Conclusión}

La ISQ post craneotomías presentó alta tasa de incidencia, con mayor afectación en mujeres y en un grupo de edad joven entre 31 a 40 años; los bacilos gramnegativos fueron los patógenos responsables de la mayoría de las ISQ.
Los factores de riesgo que presentaron mayor relación significativa con la ISQ, fueron patología tumoral, ASA I, hospitalización post cirugía prolongada, estadía en sala de recuperación más de 48 horas, ventilación mecánica, CVC, injerto dural, transfusión sanguínea, sonda vesical más de 72 horas, re intervenciones y fuga de LCR.

Según el sitio de infección, ocurrió un alarmante porcentaje de afectación en el sistema nervioso central; que además prolongó el tiempo promedio de hospitalización post infección a más de 30 días y la mitad de ellos requirieron re intervenciones, afectando principalmente la calidad de vida del paciente y posteriormente al sistema de salud, limitando aún más sus recursos.

Recomendación: Intensificar el apoyo por parte de las instituciones de salud para la realización de nuevos estudios y vigilancia activa, para crear protocolos de prevención $y$ tratamiento precoz de las infecciones postquirúrgicas.

\section{CONFLICTOS DE INTERES}

Las autoras declaran no tener ningún conflicto de interés.

\section{AGRADECIMIENTOS}

Agradecemos profundamente a la Dirección General, Jefa del Servicio de Neurocirugía y Servicio de Docencia e Investigación del Hospital Mario Catarino Rivas por permitirnos realizar el presente estudio; a los médicos especialistas de los departamentos de Neurocirugía y Anestesia por su inestimable apoyo; al personal de las salas de hospitalización, archivo y estadística por su disposición y valiosa colaboración. 


\section{REFERENCIAS BIBLIOGRÁFICAS}

1. Farrell CJ, Pisculli ML, Barker FG. Postoperative Infections of the Head and Brain. En Winn HR, Youmans Neurological Surgery. 6a ed: Elsevier; 2011. pag. 560-569.

2. Ducel G, Fabry J, Nicolle L. \& World Health Organization. Dept. of Epidemic and Pandemic Alert and Response. Prevención de las infecciones nosocomiales: guía práctica. 2a ed. Ginebra: Organización Mundial de la Salud; 2003.

Disponible en: http://www.who.int/csr/ resources/publications/ES_WHO_ CDS_CSR_EPH_2002_12.pdf.

3. Martínez V, Perdomo M, Luigi T, Ibarra B. Agentes etiológicos en infecciones post-quirúrgicas en servicios del hospital "Luis Blanco Gásperi". Carabobo, Venezuela. Salus. [Internet] 2014; 18(3): 7-14. [Citado 2 de julio 2017] Disponible en: http://www.redalyc.org/ articulo.oa?id=375939028003.

4. Augello Díaz SL, Hernández González $\mathrm{K}$, Salomón Vila AS. Infecciones nosocomiales en el postoperatorio neuroquirúrgico; CCM [Internet] 2015; 19 (3): 453-464. [citado 24 de junio 2017].

Disponible en: http://scielo.sld.cu/pdf/ ccm/v19n3/ccm07315.pdf.

5. Kaba Akoriyea S, Epidemiologia de la Infección Nosocomial en Neurocirugía, Santiago de Compostela: Universidade. Servizo de Publicacións e Intercambio Científico, 2009. ISBN 978-84-9887305-4. [citado 24 de junio 2017];

Disponible en: http://hdl.handle.net/ $10347 / 2605$.

6. Mangram A J, Horan TC, Pearson ML, Silver LC, Jarvis WR. Guideline for the prevention of surgical site infection, 1999. Infect Control Hosp Epidemiol, [Internet] 1999; 20(4): 247-264. [Citado 22 de junio 2018];

Disponible en: https://stacks.cdc.gov/ view/cdc/7160.

7. Anaya DA, Dellinger EP. Infecciones quirúrgicas y elección de antibióticos. En Townsend CM, Beauchamp RD, Evers BM, Mattox KL. Sabiston Tratado de Cirugía. 18a ed: Elsevier; 2009. P 299-327.

8. Berríos-Torres SI, Bratzler DA, et al. CDC Guide line for the Prevention of Surgical Site Infection, 2017. JAMA Surg. [Internet] 2017; 152(8):784-791. [Citado 22 de junio 2018];

Disponible en: doi:10.1001/jamasurg. 2017.0904.

9. Garmendia García F, Palmero Maestre L, Gutiérrez Crespo PP, Castillo Lara GE. Comportamiento de las infecciones en el Servicio de Neurocirugía del Hospital Clínico-Quirúrgico "Dr. Miguel Enríquez". Rev haban cienc méd. [Internet] 2018; 17(1): 39-47. [citado 21 de junio 2018];

Disponible en: http://www.revhabanera. sld.cu/index.php/rhab/article/view/2206. pdf.

10. Korol E, Johnston K, Waser N, Sifakis F, Jafri HS, Lo M. et al. A Systematic Review of Risk Factors Associated with Surgical Site Infections among Surgical Patients. PLoS ONE [Internet] 2013; 8(12): e83743. [Citado 22 de junio 2018];

Disponible en: http://journals.plos.org/ plosone/article/file?id=10.1371/journal. pone.0083743\&type=printable. 
11. Cheng $\mathrm{H}$, Chen BP, Soleas IM, Ferko NC, Cameron CG, Hinoul P. Prolonged Operative Duration Increases Risk of Surgical Site Infections: A Systematic Review. Surgical Infections. [Internet] 2017; 18(6):722-735. [Citado 22 de junio 2018];

Disponible en: https://www.ncbi.nlm.nih. gov/pmc/articles/PMC5685201/pdf/sur 2017.089.pdf.

12. Taha MM, Abouhashem S, Abdel-Rahma AY. Neurosurgical Wound Infection in Egypt. Turkish Neurosurgery. [Internet] 2014; 24(1):8-12. [Citado 15 de junio 2018];

Disponible en: http://www.turkishneuro surgery.org.tr/pdf/pdf_JTN_1245.pdf .

13. Jiang X, Ma J, Hou F, LI J, LI R, Lang H. Neurosurgical Site Infection Prevention: Single Institute Experience. Turk Neurosurg [Internet] 2016; 26(2): 234-239. [Citado 22 de junio 2018];

Disponible en: https://pdfs.semantics cholar.org/45eb/164a6824b226ca47fbd 0c10a8c190f6b41f1.pdf.

14. Buang SS, Haspani MS. Risk factors for neurosurgical site infections after a neurosurgical procedure: a prospective observational study at Hospital Kuala Lumpur. Med J Malaysia. [Internet] 2012; 67(4):393-398. [Citado 22 de junio 2018];

Disponible en: http://www.e-mjm.org/ 2012/v67n4/Surgical-site-infection.pdf.

15. Petric A, lonac M, Brinzeu C, Brinzeu A. Surgical site infections surveillance in neurosurgery patients. TMJ [Internet] 2009; 59(4): 339-343. [Citado 24 de junio 2017];

Disponible en: http://www.tmj.ro/pdf/ 2009_number_3_4_8634616731273 93.pdf.

16. Lietard C, Thébaud V, Burnichon G,
Besson G, Lejeune B. Comparative Analysis of 75th Percentile Durations for Neurosurgical Procedures in France and in US National Noscomial Infection Surveillance System Data. Infection control and hospital epidemiology January. [Internet] 2008; 29(1): 73-75. [Citado 24 de junio 2017];

Disponible en: https://www.researchga te.net/publication/5678967.

17. Korinek AM, Baugnon T, Golmard JL, Effenterre Rv, Coriat P, Puybasset L. Risk factors for adult nosocomial meningitis after craniotomy role of antibiotic prophylaxis; Neurosurgery [Internet] 2006 58(7): 126-133. [Citado 24 de junio 2017];

Disponible en: http://www4.utsouthwes tern.edu/swneurosurg/ll_-_Risk_Factors _for_Adult_Nosocomial_Meningitis.pdf.

18. Buffet-Bataillona S, Haegelenb C, Riffaudb L, Bonnaure-Malletc M, Brassierb G, Cormiera M. Impact of surgical site infection surveillance in a neurosurgical unit. Journal of Hospital Infection. [Internet] 2011; 77(4): 352-355. [Citado 24 de junio 2017];

Disponible en: https://www.journalofhos pitalinfection.com/article/S0195-6701 (10)00466-4/pdf.

19. Martínez Checa J, Planes Martínez I, González de la Flor P, Fernández Sierra $M . A$, Jiménez Romano $E$, Cordero Moreno A. Incidencia y factores de riesgo de infección nosocomial de herida quirúrgica en Neurocirugía. Neurocirugía [Internet] 2000; 11: 103-109. [Citado 4 de julio 2017];

Disponible en: https://www.sciencedi rect.com/science/article/pii/S11301473 00707445.

20. Chisbert Genovés MP, Moreno Oliveras L, Ballo Martínez N, Piquer Belloch J. Infección de la herida quirúrgica en neu- 
rocirugía, análisis de los factores de riesgo. Cátedra de Neurociencias CEU - NISA. [Internet] 2013 [Citado 28 de marzo 2018].

Disponible en: https://www.catedraneu rocienciascnn.com/wp-content/uploads/ TRABAJO-COMPLETO.-REFERENCIA -41 .pdf.

21. Chiang HY, Kamath AS, Pottinger JM, Greenlee JD, Howard MA, Cavanaugh $\mathrm{JE}$, et al. Risk factors and outcomes associated with surgical site infections after craniotomy or craniectomy. J Neurosurg. [Internet] 2014; 120(2):509-521. [Citado 22 de junio 2018];

Disponible en: http://thejns.org/doi/full/ 10.3171/2013.9.JNS13843.

22. Sánchez-Arenas R, Rivera-García BE, Grijalva-Otero I, Juárez Cedillo T, Martínez-García MC, Rangel-Frausto S. Factors associated with nosocomial surgical-site infections for craniotomy in Mexico City Hospitals. Cir Ciruj. [Internet] 2010; 78(1): 5-13. [Citado 24 de junio 2017];

Disponible en: http://www.medigraphic. com/pdfs/circir/cc-2010/cc101b.pdf.

23. Bellusse GC, Ribeiro JC, Campos FR, Poveda BV, Galvão CM. Fatores de risco de infecção da ferida operatória em neurocirurgia. Acta Paul Enferm. [Internet] 2015; 28(1): 66-73. [Citado 24 de junio 2017];

Disponible en: http://www.scielo.br/pdf lape/v28n1/en_1982-0194-ape-028 -001-0066.pdf.
24. Perdomo - Sabillón NM, Nieto TR. Infecciones del sitio quirúrgico en craniotomías: ¿qué antibióticos profilácticos hay que usar en nuestro medio?.Revista Médica de los Post Grados de Medicina UNAH [Internet] 2006; 9(3): 365-372. [Citado 24 de junio 2017];

Disponible en: http://65.182.2.242/RMP/ pdf/2006/pdf/Vol9-3-2006-12.pdf.

25. Múñez E, Ramos A, Álvarez de Espejo T, Vaqué J, Sánchez-Payá J, Pastor V. et al. Etiología de las infecciones quirúrgicas en pacientes sometidos a craneotomía. Sociedad Española de Neurocirugía. [Internet] 2012; 23(2): 54-59. [Citado 12 de junio 2018];

Disponible en: http://neurocirugia.else vier.es/es-pdf-S1130147312000395.

26. Palacios-Saucedo GC, Garza-Camargo M, Briones-Lara E, Carmona-González S, García-Cabello R, Islas-Esparza LA. et al. Evaluación del uso de antibióticos e impacto de una intervención dirigida a modificar la conducta prescriptiva en profilaxis quirúrgica en 6 hospitales del área metropolitana de Monterrey. Cirugía y Cirujanos. [Internet] 2016 85(6): 459-470. [Citado 15 de junio 2018];

Disponible en: http://dx.doi.org/10.1016/ j.circir.2016.10.033. 